\title{
MS21-P02 | ELECTRON DENSITIES OF MOLECULAR CRYSTALS FROM POWDER X-RAY
}

\section{DIFFRACTION}

Svane, Bjarke (Aarhus University, Aarhus C, DNK); Brummerstedt Iversen, Bo (Aarhus University, Aarhus, DNK)

Detailed knowledge of the nature of the chemical bonding is a prerequisite for understanding the physical and chemical properties of materials. This information is best available in the electron density (ED). Virtually all experimental ED distributions are determined from structure factors extracted from single crystal X-ray diffraction, since this has been regarded the optimal way to obtain data of the highest quality. However, our recent work has shown that data obtained from powder X-ray diffraction (PXRD) can exceed the data quality from single crystal diffraction [1-5].

I aim to determine ED distributions and atomic displacement parameters (ADPs) of molecular materials from highly accurate PXRD data using the well-known Hansen-Coppens multipole modelling [6]. Structure factors are obtained from Rietveld refinement to partition intensity between overlapping reflections. The modelling is improved using aspherical approximate atomic scattering factors. The better model will lead to more accurate intensity extraction, which can then be used in a subsequent full ED refinement using the multipole method. This is of critical importance if the PXRD method to obtain EDs is to be extended from small unit cell inorganic solids to molecular crystals with severe peak overlap.

[1] N. Bindzus et al. (2014), Acta Cryst. A

[2] T. Straasø et al. (2014), J. Synchrotron Rad.

[3] N. Wahlberg et al. (2015), J. Phys. Chem.

[4] K.Tolborg et al. (2017), Acta Cryst. B

[5] M. R. V. Jørgensen et al. (2014), IUCrJ

[6] Hansen, N. K. \& Coppens, P. (1978). Acta Cryst. A 